\title{
Virtualization of Wireless Sensor Networks
}

\author{
Ali Kashif Bashir \\ Advanced Network Architecture Laboratory, Graduate School of Information Science and Technology, Osaka University, Japan \\ *Corresponding author: Ali Kashif Bashir, Advanced Network Architecture Laboratory, Graduate School of Information Science and Technology, Osaka University, \\ 565-0871, Japan, Tel:+81668794542; Fax: +81668794544; Email: ali-b@ist.osaka-u.ac.jp
}

Rec date: Aug 26, 2015, Acc date: Aug 27, 2015, Pub date: Aug 31, 2015

Copyright: $\odot 2015$ Bashir AK, This is an open-access article distributed under the terms of the Creative Commons Attribution License, which permits unrestricted use, distribution, and reproduction in any medium, provided the original author and source are credited.

\section{Editorial}

Internet-of-Things (IoT) is considered one of the next technological revolutions. It enables communication among objects of various kinds. Wireless Sensor Networks (WSNs), being the basic elements of IoT, can help users (humans or machines) to interact and react to realworld events. WSN nodes are capable of sensing, communications, and computations; and are getting more powerful. Though various advantages, but one obvious drawback WSNs have is that they are domain-specific and task-oriented, which means they are designed to serve one particular application without the possibility of being reused for another application. This leads us to redundant deployment of WSN infrastructure in a particular are for multiple similar applications. For example, in a military environment the temperature monitoring WSN infrastructure would probably be different from the infrastructure to monitor the enemy movement. Such redundancy increase infrastructure cost. With the philosophy of IoT, in the future, WSN deployment should support multiple applications simultaneously.

Virtualization is an established concept that allows multiple applications to run concurrently on a single physical infrastructure. It creates the abstraction of actual physical computing resources into logical units. Virtualization is a key technique for the realization of the Future Internet and is widely used in data centers/clouds. Adopting virtualization in WSN, can allow the efficient utilization of deployments, as multiple applications can co-exist on a single virtualized WSN. It can also create multiple logical WSNs on top of a single physical WSN where physical WSN will be called virtualized and logical ones, virtual. Virtualization eliminates the tight coupling between WSN services/applications and WSN deployments. This will provide equal opportunities to experience as well as novice application developers to develop innovative applications on the existing deployments. Another advantage of virtualization is that it helps in defining a business model by introducing many new business entities such as physical WSN provider, virtual network provider, virtual network operator and the service providers. Such expansions will increase commercialization and increase the revenue in the WSN technology.

To understand this WSN virtualization, consider an infrastructure of WSN that is deployed to monitor the volcanic activity of a mountain by the disaster management administration. Instead of deploying another WSN for another application, for example, local city management to monitor the land sliding or wildlife department to monitor the animal activities, the already existing WSN infrastructure can be virtualized, fully or partially, to accommodate the new applications. The concept of WSN virtualization is applied to several interesting application areas. For example, mobile crowd sensing takes advantage of virtualizing these sensors through participatory and opportunistic sensing. Sensing-as-a-Service (SaaS) model is presented in along with use case scenarios. WSN virtualization can help realize the SaaS model through cost-efficient utilization of deployed sensors.

Virtualization can be discussed in two aspects: node-level virtualization and network-level virtualization. The first one enables multiple applications to run their tasks concurrently on a single sensor node, so that a sensor node can become multi-purpose. The basic concept of node-level virtualization is illustrated in figure 1 (a). Network-level virtualization is a quite broad term, as one network can be virtualized in several ways. In general, a virtual wireless sensor network (VWSN) is a logical subset of the underlying WSN deployed with a particular application to run their protocols. One simple illustration of VWSN is given in figure 1 (b) where a single VSWN is virtualized into two virtual WSNs. However, with network-level virtualizations one application can deploy their VSWN on top of more than one WSNs as well.

Virtualization of WSNs is an emerging research area. With the expected expansion in its business model, we expect a huge growing interest of application developers in this area. Journal of Sensor Networks and Data communication welcomes articles in WSN virtualizations. We plan to publish latest and groundbreaking research in every aspect of WSN virtualization. Some of these aspects are given below and these points are also the research issues/directions in WSN virtualization.

- Virtual node/network embedding algorithms

- New business models for: Infrastructure-as-a-service, Sensing-asa-service, node-as-a-service

- Service composition in the virtualized environments

- Resource allocation

- VSWN for cloud applications/services

- Advanced virtual architectures and frameworks

- Energy efficient virtual solutions

- Service orchestration and provisioning

- Bandwidth allocation and load-balancing

- New protocols and simulation tools 
Citation: Ali Kashif Bashir (2015) Virtualization of Wireless Sensor Networks . Sensor Netw Data Commun 4: e105. doi: 10.4303/2090-4886.1000e105

Page 2 of 2

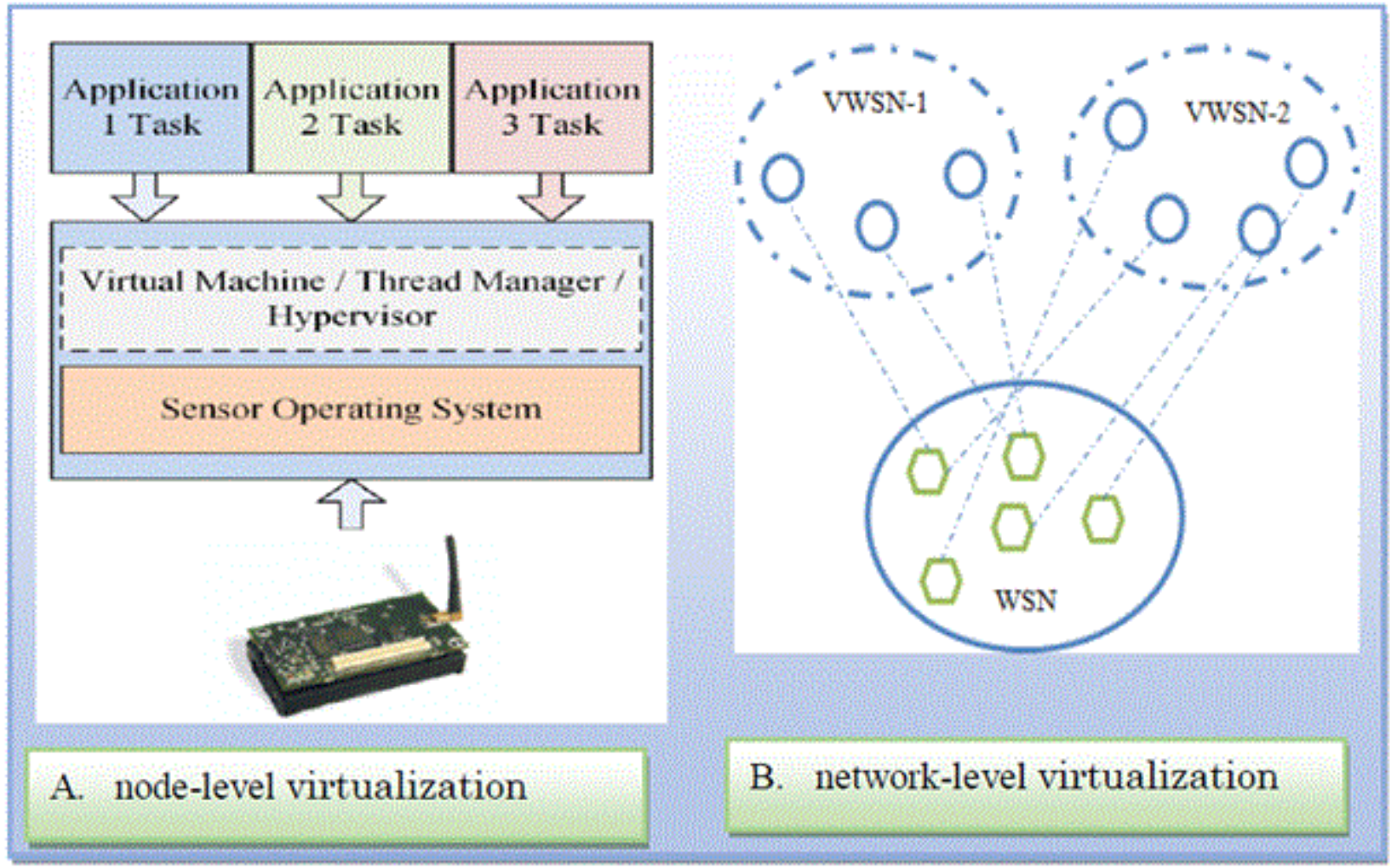

Figure 1: Virtualization of WSNs. 